\title{
Early prediction of language and literacy problems: Is 18 months too early?
}

Fiona J Duff, Kate Nation, Kim Plunkett, Dorothy V M Bishop

There is a lack of stability in language difficulties across early childhood: most late talkers (LTs) resolve their difficulties by pre-school; and a significant number of children who were not LTs subsequently manifest language difficulties. Greater reliability in predicting individual outcomes is needed, which might be achieved by waiting until later in development when language is more stable. At 18 months, productive vocabulary scores on the Oxford Communicative Developmental Inventory were used to classify children as LTs or average talkers (ATs). Thirty matched-pairs of LTs and ATs were followed up at school-age (average age 7 years), when language and literacy outcomes were assessed. For 18 children, intermediate testing at age 4 had classified them as showing typical development (TD) or specific language impairment (SLI). After correcting for multiple comparisons, there were no significant differences between the LTs and ATs on any outcome measure, and the LTs were performing in the average range. However, there were large-sized effects on all outcomes when comparing the TD and SLI groups. LT status on its own is not determinative of language and literacy difficulties. It would therefore not be appropriate to use expressive vocabulary measures alone to screen for language difficulties at 18 months. However, children with language impairment at age 4 are at risk of enduring difficulties. 
Fiona J. Duff

Kate Nation

D.V.M. Bishop

11 
15 The term 'late talkers' (LTs) generally refers to young children aged 18- to 35-months who are slow to develop spoken language in the absence of any known primary cause (Rescorla, 2011). Prevalence rates for LTs differ according to the inclusion criteria and the population sampled, but a recent study of a large community sample suggested that as many as $20 \%$ of young children can be classified as LTs (Reilly et al., 2007). A key question is whether LTs should be a cause for concern; here there can be a mismatch between views of academic researchers and those concerned with policy. Research evidence shows that there is a lack of stability in language across early childhood development - particularly when measured by vocabulary - making prediction of outcomes from infancy unreliable. For example, while the early language difficulties of some LTs persist into childhood, the majority of LTs perform in the average range by pre-school (Rescorla, 2011). Moreover, a significant number of children show late emerging language delay: that is, children who were not originally classified as LTs go on to exhibit language difficulties in the pre-school years (e.g., Dale et al., 2003; Henrichs et al., 2011). It is also important to consider the longerterm outcomes of LTs, given the proposal that children whose language skills appear to have normalised will in fact manifest language and literacy difficulties later in development - so called illusory recovery (Scarborough \& Dobrich, 1990; but see Dale et al., 2014). In fact, the majority of LTs perform in the average range on language and literacy measures in the later primary school years and beyond, though often at a level significantly below that of their unaffected peers (e.g., Dale \& Hayiou-Thomas, 2013; Paul et al., 2007; Rescorla, 2002, 2005, 2009; Rice, Taylor, \& Zubrick, 2008). In the main, then, the language difficulties of most LTs are short-lived. 
Nevertheless, outside academia, there are those who claim that LTs should be identified and supported early on. The logic is that by intervening early, there is a better chance of avoiding the worst outcomes and of making effective changes while

41 the brain is still plastic. This viewpoint is articulated by the C4EO early intervention 42 group (2010), who note that many children with language delay catch up with their 43 peers, but assert that this is because they have had "the right support". This is rather misleading, since most studies report good outcomes despite lack of intervention. This is a critical point, because if there is spontaneous improvement in LTs, then early intervention is not warranted, especially if it takes funds from other deserving causes. Unfortunately, spontaneous improvement can also reinforce the misapprehension surrounding outcomes of LTs: if these children are identified and provided with intervention, they then improve, and the improvement is attributed to the intervention. The lack of treatment effect is only evident if one studies an untreated group of LTs, as was done in the study by Wake et al. (2011). Nevertheless, the view remains among some of those influencing policy that a child's vocabulary level at or before 2 years of age can be used to predict language and pre-literacy skills at school entry (e.g., Roulstone et al., 2011).

An important goal, then, is to be able to distinguish better between early language difficulties that will resolve versus persist - that is, to improve prediction of outcomes at an individual level. This might be achieved by waiting until later in development, once language may have become more stable, or by identifying better predictors early on. Here, we take the former approach. We report a follow-up

60 investigation of a sample of children who were identified as LTs at 18 months old, and 
61 of their peers who were classed as average talkers. Our first aim was to compare the

62 language and literacy outcomes of these groups of children. Based on previous

63 findings, we hypothesised that the LTs' school-age language and literacy skills would

64 be in the average range, but fall significantly below those of their unaffected peers.

65 Information regarding language status at age 4 (typical development vs. specific

66 language impairment) was available for a subsample of children, allowing us to

67 explore a second hypothesis that language status at 4 years would be more indicative of

68 reading and language outcomes than language status at 18 months.

\section{Method}

71 Participants investigating language and literacy development (Duff, Reen, Plunkett, \& Nation, 2015). Informed written parental consent was given for all participants, and ethical approval was granted by the University of Oxford's Central University Research Ethics Committee (MSD/IDREC/C1/2012/56). For the main project, 300 children whose vocabularies had been measured in infancy (between 16 and 24 months) were followed-up at school-age, when they were aged between 4 and 9 years old. Though age was allowed to vary at both test points, it was accounted for in the statistical analyses.

In the present report, we followed earlier investigations (e.g., Bishop et al., 2012) by focusing on those children whose vocabulary was measured at $18-19$ months

$83(\mathrm{~N}=153)$. These children were subsequently classified either as 'late talkers' (LTs) or 
'average talkers' (ATs). Classification procedures followed that of Bishop et al. (2012) and used data from the Oxford Communicative Development Inventory (OCDI; Hamilton, Plunkett, \& Schaffer, 2000). This Anglicised adaptation of the American CDI (Fenson et al., 1994) comprises 416 words, and parents were required to indicate which of these words their child was able to understand (comprehension) and understand and say (production). The first 12 items, which are instances of onomatopoeia, were excluded from analyses leaving a total of 404 items. Children were categorised as LTs if their raw OCDI productive vocabulary score at 18 or 19 months of age $(t 1)$ was 10 words or fewer. In the sample used by Bishop et al. (2012) this equated to performance levels at least $1 \mathrm{SD}$ below the mean (see https://osf.io/t35af/). However, for the current, slightly younger sample, this cut-off corresponded to the $25^{\text {th }}$ centile for the norms of Hamilton et al. (2000), whereas a cutoff of six words or less corresponded to the $16^{\text {th }}$ centile (i.e., equivalent to one SD below the mean). We used the more liberal criterion of 10 words or fewer for this study, but in the analysis, we consider the extent to which the inclusion of children with only mild delays affects the findings. Thirty ( 22 boys) of the 153 children were classified as LTs $(20 \%)$ using the 10-word cut-off, and twenty of these met the more stringent cut-off of six words or fewer. Following Bishop et al. (2012), ATs were those children whose OCDI production raw scores fell between 14 and 65 out of 404; this corresponds to the $31^{\text {st }}$ to $73^{\text {rd }}$ centile on the norms for 18 - to 19 -month-olds from Hamilton et al. (2000). This yielded 66 children, of whom 30 were matched pairwise to the LTs, based on gender and age at $t 2$ (average absolute difference in age $=1.33$ months, range $=0-3$ months). 
OCDI scores at $t 1$ are reported in Table 1 , both for the full sample of 30 pairs, and for the 20 pairs with a child meeting the more severe cut-off of 6 words or fewer. Paired-samples $t$-tests revealed that the groups did not differ on vocabulary comprehension; although there was a trend for lower comprehension when a stricter cut-off for LTs was used. Note, however, that the mean comprehension scores were virtually identical for the 20 strictly-defined LTs and the 30 selected by the laxer criterion. Demographic information at $t 1$ and $t 2$ is also given in Table 1. Children ranged in age from 4 to 9 years at $t 2$, but owing to the matching procedure, there was no group-level difference in age. The Index of Multiple Deprivation (IMD) was calculated based on postcode data to give an indication of socioeconomic status (SES). The IMD returns rank-ordered data, ranging from 1 (high deprivation) to 32,482 (low deprivation). Both groups have IMD scores higher than the national average $(16,241)$ but similar to the average for their local county of Oxfordshire $(21,809)$ (Department for Communities and Local Government, 2011). IMD did not differ significantly between the two groups at either time point. assessed at age 4 as part of a separate study by Bishop et al. (2012). At that time point,

124 children were categorised according to whether or not they reached criteria for Specific

125 Language Impairment (SLI). Full details are given in Bishop et al. (2012). Briefly, 126 children were identified as having SLI if their performance was impaired on at least 127 two language measures, but their nonverbal IQ was in the average range $(\geq 85)$. Children were classified as having typical development (TD) if no more than one 129 language measure was impaired, and their nonverbal IQ was in the average range. In 
130 both the AT and LT groups, 3 children were classified as having SLI and 6 children as

131 TD $^{1}$

132 Measures

133 Vocabulary knowledge. The Receptive and Expressive One Word Picture

134 Vocabulary Tests (Brownell, 2000) were administered. To assess receptive

135 vocabulary, children heard a series of graded words, and were asked to select the

136 corresponding picture from four alternatives for each word (test/re-test reliability $=.78$

137 to .93). For expressive vocabulary, children were required to name a series of graded

138 pictures (test/re-test reliability $=.88$ to .91$)$.

139 Phonological short-term memory. Measures of nonword and sentence

140 repetition tapped short-term memory for verbal information. On the Children's Test of

141 Nonword Repetition (Gathercole \& Baddeley, 1996), children repeated aloud 40

142 individual nonwords, ranging from two to five syllables in length (test/re-test reliability

$143=.72)$. For the Recalling Sentences subtest of the Clinical Evaluation of Language

144 Fundamentals (CELF-III UK - Semel, Wiig, \& Secord, 2000), children were required

145 to repeat orally presented sentences of increasing length and grammatical complexity

$146 \quad$ (test/re-test reliability $=.93$ to .94$)$.

147 Phonological awareness. The Elision subtest of the Comprehensive Test of

148 Phonological Processing (Wagner, Torgesen, \& Rashotte, 1999) was administered.

149 For each orally presented word, children were asked to delete a sublexical unit

\footnotetext{
${ }^{1}$ The rate of ATs with SLI in the current study is higher than expected, possibly due to sampling bias, if parents whose children had language problems were more willing to consent to the follow-up. In the original study with a larger sample, the rates were $29 \%$ of LTs and $14 \%$ of ATs (see Bishop et al., 2012).
} 
150 (syllable or phoneme) and supply the word that remained (test/re-test reliability $=.79$

151 to .88$)$.

152 Reading accuracy. For the Diagnostic Test of Word Reading Processes

153 (Forum for Research into Language and Literacy, 2012), children read aloud lists of

154 graded nonwords, regular words and exception words (reliability, $\alpha=.99$ ).

155 Reading comprehension. Passage reading comprehension was assessed in

156 children aged 5 upwards via the York Assessment of Reading Comprehension

157 (Snowling et al., 2009). Children read aloud two short stories and after each story

158 answered a series of eight related questions (reliability, $\alpha=.48$ to .77 ).

159 Nonverbal ability. Nonverbal reasoning was assessed via the Matrices subtest

160 of the British Abilities Scale II (Elliot, Smith, \& McCulloch, 1997). Children were

161 presented with an incomplete matrix of abstract figures and were instructed to choose

162 the correct shape from an array of six to complete the matrix (test/re-test reliability $=$ $163.64)$

164 Procedure

165 For the follow-up assessments at $t 2$, children were seen individually by a 166 member of the research team. Assessment sessions lasted approximately 1 hour and 167 were conducted at school, home, or the Department of Experimental Psychology, 168 University of Oxford.

\section{Results}

The scores for the ATs and LTs at follow-up ( $t 2)$ are reported in Table 2. The

172 standardised scores show that the AT group is performing in the average to above 
173

174

175

176

177

178

179

180

181

182

183

184

185

186

187

average range on all outcomes. For the full sample of 60 children, the maximum

number achieving scores below average ( $>1 \mathrm{SD}$ below the normative mean) on any given measure is $4(13 \%)$. The LT group performed in the average to high-average range on all outcomes, with no more than 3 children (10\%) achieving below average scores on any one measure. For statistical analysis, we used raw scores with age regressed out of them (on the basis of the entire dataset of $\mathrm{N}=300$ from Duff et al., 2015). This gives scores that are highly correlated with the standardised scores but with greater precision. (To facilitate readability of Table 2, these means are not included; the data are available in the Supporting dataset).

There is a trend for the LTs to have lower scores on most measures (apart from phonological elision and nonverbal IQ). Matched-pairs $t$-tests were performed on the age-regressed scores to assess whether there were any significant differences between the groups. Effect sizes (Cohen's $d$ ) were calculated for each contrast by dividing the difference in group means by the pooled standard deviation; $d$ s of $0.2,0.5$ and 0.8 represent small, medium and large effects, respectively (Cohen, 1992). Concerning the sample of 30 LTs and their matched ATs, there was a significant medium-sized effect on expressive vocabulary. However, after correcting for multiple comparisons using the Benjamini-Hochberg procedure (Benjamini \& Hochberg, 1995), this difference was no longer significant. There was no effect of group on any of the remaining language, literacy, or nonverbal measures.

All analyses were repeated using just the 20 LTs with OCDI production scores of 6 words or fewer, and their matched ATs. As can be seen in Table 2, this tended to give greater effect sizes, but did not have a material effect on the pattern of results. 
Once again, the only difference reaching the .05 level of significance was on expressive vocabulary, and this did not survive Benjamini-Hochberg correction for multiple comparisons. The similarity of results with the two cut-offs suggests that the severity of the initial expressive language delay is not related to the extent of language deficit at t2. To check this impression further, Pearson correlations were computed between the 11 OCDI production score of the LT member of a pair and the difference between pair members on each of the $\mathrm{t} 2$ variables from Table 2 . None of the correlations was significant at the .05 level.

We turn now to the subsample of $9 \mathrm{AT} / \mathrm{LT}$ matched pairs who had previously been assessed at age 4 . At $t 2$ in the present study, they were on average aged 8 years, 9 months (range $=8 ; 01$ to 9;04). Contrasts between this subsample of ATs and LTs were similar to those for the whole sample: there were no significant differences (all $p \mathrm{~s}$ $>.20)$.

Table 3 shows how LI status at 4 years relates to outcomes at $t 2$. The TD group performed in the average to above average range on all outcomes, while the SLI group performed in the below average to high-average range. Comparison of age-regressed scores across the two groups revealed large-sized effects on all outcome measures, favouring the TD group. According to independent samples $t$-tests, the group effect was significant (even after correcting for multiple comparisons) for expressive vocabulary, nonword repetition, recalling sentences, reading accuracy and reading comprehension. The group effect was not significant for receptive vocabulary, phonological elision, or nonverbal IQ. 
We investigated the school-age outcomes of a group of children defined as LTs at age 18 months, a subsample of whom had also been assessed for SLI at age 4 years. This enabled us to test the hypotheses that the subsequent language and literacy skills of LTs would be in the average range for their age, but fall below the level of their unaffected peers (ATs); and that language status at 4 years would be more indicative of outcomes than language status at 18 months. average range on all language, literacy and nonverbal measures - with very few individuals reaching criterion for an impairment. In fact, there were no statistically significant differences between the LTs and ATs on any of the outcomes, and all contrasts reflected small effect sizes - except on expressive vocabulary where there was a medium-sized effect in favour of the ATs. Overall, then, we found no evidence for subclinical problems in this group of LTs. We considered whether this null result might be due to use of a lax cut-off for LTs of 10 words or fewer on OCDI Production. However, results were virtually identical when analysis was confined to the 20 LTs with more serious expressive delays, with six words or fewer at t1. Furthermore, the severity of vocabulary delay at $\mathrm{t} 1$ was unrelated to the size of difference between LTs and their matched AT controls at $\mathrm{t} 2$. Note, however, that we did not include a measure of grammatical ability at 2 ; thus, it remains possible that weaknesses may have been detected in this area of language. 
242 language and literacy outcomes at 7 years of age, SLI status at 4 years did. Children

243 with SLI went on to have lower scores on all outcome measures compared to the TD

244 children, and the magnitude of the differences reflected large-sized effects. Despite the

245 low power from the small sample size, differences were statistically significant for

246 expressive vocabulary, nonword repetition, recalling sentences, reading accuracy and

247 reading comprehension (but not receptive vocabulary, phonological elision, or

248 nonverbal IQ). Moreover, the differences were educationally significant, with the SLI

249 subgroup performing 20 standard score points below the TD subgroup on expressive

250 vocabulary and 26 points below on reading accuracy.

251 Our findings have added to the literature which shows that LT status on its

252 own, defined on the basis of parent-reported expressive vocabulary, is by no means

253 determinative of language and literacy difficulties (e.g., Dale \& Hayiou-Thomas, 2013;

254 Paul et al., 1997), that parent report of expressive vocabulary in infancy is not a

255 reliable indicator of outcomes (e.g., Dale et al., 2003), and that language skills - as

256 measured by vocabulary - are not stable across infancy into childhood (e.g., Duff et al.,

257 2015; Ghassabian et al., 2013; Reilly et al., 2010). It follows that it would not be

258 appropriate to use expressive vocabulary measures alone to screen for language

259 difficulties in infancy. Our results also suggest that presence of a language impairment

260 at age 4 years is a much better indicator of enduring difficulties than being an LT at

261 age 18 months. In any time series, one expects to see stronger correlations between

262 adjacent time points than between more remote points, and to some extent this may

263 account for the better prediction of outcome from later ages. However, this cannot

264 explain why prediction is better from, say, 4 to 5 years, as opposed to prediction from 2 
265 to 3 years. To account for that, it seems necessary to invoke the idea that whatever

266 causes persistent language impairment can be distinguished from the factors

267 determining the age at which the child starts to rapidly acquire words. There appears

268 to be a wide range of normal variation in the latter process which can be seen as part of

269 maturation rather than reflective of any disorder. The older a child is, the lower the

270 probability that poor language is just due to normal maturational variation.

A question of interest concerns the optimal age to identify children at risk for

272

persistent language difficulties: given that prediction is poor at 18 months and good at

4 years, we may ask whether there is a step change in predictive utility of language

assessment. Dollaghan and Campbell (2009) found that children with a vocabulary

deficit at 4 years had a significantly increased risk for manifesting a vocabulary deficit

at 6 years, while a deficit at 3 years was not associated with a later impairment. This

provides some indication that prediction of outcomes improves after age 3; however,

the authors were cautious about their results, as a slight change to the criterion for a

vocabulary deficit rendered the elevated risk of persistent deficits from 4 to 6 years

nonsignificant. A recent study by Dale and Hayiou-Thomas (2013) showed that the

at age 3 rather than at age 2 . However, these difficulties were still only apparent in a

subsequently manifest an impairment was poor. This suggests that even prediction of 
288 Furthermore, the LT group was selected on the basis of expressive vocabulary, without 289 regard to language comprehension (in-keeping with the classic definition of LTs). In

290 the full sample there was a non-significant trend for the LTs to have lower vocabulary

291 comprehension at 18 months. It is likely that a delay in early vocabulary development

292 might assume more importance in children whose development is compromised by

293 other risk factors. Various risks have been shown to be additive to that associated with

294 late-talking, for example, male gender, receptive language difficulties, and family

295 history of language or literacy difficulties (e.g., Law et al., 2012; Ghassabian et al.,

296 2013; Reilly et al., 2010). Nevertheless, to date, models that incorporate such variables

297 have failed to explain enough variance in language outcomes to be usefully predictive

298 at an individual level. A major goal for future research is to generate models that can

299 discriminate reliably between transient versus persistent early language delay; and

300 these models must be simple enough to be useful clinically. Our study, however,

301 suggests that a more fruitful approach may be to conduct longitudinal studies that

302 measure language from infancy into the later primary years, perhaps annually, to

303 determine at what point in development language becomes stable enough for reliable

304 prognoses to be made. 
307 Benjamini Y, Hochberg Y. 1995. Controlling the false discovery rate: A practical and powerful approach to multiple testing. Journal of the Royal Statistical Society 57:289-300.

Bishop DVM, Holt G, Line E, McDonald D, McDonald S, Watt H. 2012. Parental phonological memory contributes to prediction of outcome of late talkers from 20 months to 4 years: A longitudinal study of precursors of specific language impairment. Journal of Neurodevelopmental Disorders 4:3.

Bowyer-Crane C, Snowling MJ, Duff FJ, Fieldsend E, Carroll JM, Miles JNV, Hulme C. 2008. Improving early language and literacy skills: Differential effects of an oral language versus a phonology with reading intervention. Journal of Child Psychology \& Psychiatry 49:422-432.

Brownell R. 2000. Expressive and Receptive One Word Picture Vocabulary Tests $\left(2^{\text {nd }}\right.$ Edition). Academic Therapy Publications, Novato: California.

C4EO Early Intervention Expert Group. 2010. Grasping the nettle: Early intervention for children, families and communities. London: C4EO.

Cohen, J. (1992). A power primer. Psychological Bulletin, 112, 155-159.

Dale PS. Hayiou-Thomas ME. 2013. Outcomes for late talkers. In LA Rescorla \& PS Dale, eds. Late talkers: Language development, interventions, and outcomes. Baltimore, MD: Brookes Publishing, 241-257.

Dale PS, McMillan AJ, Hayiou-Thomas ME, Plomin R. 2014. Illusory recovery: Are recovered children with early language delay at continuing elevated risk? American Journal of Speech-Language Pathology 23:437-447. 
329

330

Dale PS, Price TS, Bishop DVM, Plomin R. 2003. Outcomes of early language delay 1. Predicting persistent and transient language difficulties at 3 and 4 years. Journal of Speech, Language, and Hearing Research 46:544-560.

Department for Communities and Local Government. 2011. English indices of deprivation 2010: Overall. Available at https://www.gov.uk/government/publications/english-indices-of-deprivation2010 (accessed 20 August 2014).

Dollaghan CA, Campbell TF. 2009. How well do poor language scores at ages 3 and 4 predict poor language scores at age 6? International Journal of SpeechLanguage Pathology 11:358-365.

Duff FJ, Reen, G, Plunkett K, Nation K. 2015. Do infant vocabulary skills predict school-age language and literacy outcomes? Journal of Child Psychology and Psychiatry.

Elliot CD, Smith P, McCulloch K. 1997. British Ability Scales II. Windsor: NFER Nelson.

Fenson L, Dale PS, Reznick JS, Bates E, Thal, DJ, Pethick SJ. 1994. Variability in early communicative development. Monographs for the Society for Research in Child Development 59:1-185.

Forum for Research into Language and Literacy. 2012. Diagnostic Test of Word Reading Processes. London: GL Assessment.

Gathercole SE, Baddeley AD. 1996. The Children's Test of Nonword Repetition. London: Psychological Corporation. 
351 Ghassabian A, Rescorla L, Henrichs J, Jaddoe VW, Verhulst FC, Tiemeier, H. 2013. Early lexical development and risk of verbal and nonverbal cognitive delay at school age. Acta Paediatrica 103:70-80.

Hamilton A, Plunkett K, Schafer G. 2000. Infant vocabulary development assessed

Paul R, Murray C, Clancy K, Andrews D. 1997. Reading and metaphonological

Law J, Rush R, Anandan C, Cox M, and Wood R. 2012. Predicting language change between 3 and 5 years and its implications for early identification. Pediatrics

Henrichs J, Rescorla L, Schenk JJ, Schmidt HG, Jadooe VWV, Hofman A, Raat H, Verhulst FC, Tiemeier H. 2011. Examining continuity of early expressive vocabulary development: The Generation R study. Journal of Speech, Language, and Hearing Research 54:854-869. 130:E132-E137. outcomes in late talkers. Speech-Language Pathology Faculty Publications. Paper 55.

Poll GH, Miller CA. 2013. Late talking, typical talking, and weak language skills at middle childhood. Learning and Individual Differences 26:177-184.

Reilly S, Wake M, Bavin EL, Prior M, Williams J, Bretherton L, Eadie P, Barrett Y, Ukomunne OC. 2007. Predicting language at 2 years of age: A prospective community study. Pediatrics 120:e1441-1449. 
372 Reilly S, Wake M, Ukoumunne OC, Bavin E, Prior M, Cini, E, Conway L, Eadie P,

373 Bretherton L. 2010. Predicting language outcomes at 4 years of age: Findings

374 from early language in Victoria study. Pediatrics 126:e1530-e1537.

375 Rescorla L. 2002. Language and reading outcomes to age 9 in late-talking toddlers.

376 Journal of Speech, Language, and Hearing Research 45:360-371.

377 Rescorla L. 2005. Age 13 language and reading outcomes in late-talking toddlers.

$378 \quad$ Journal of Speech, Language, and Hearing Research 48:459-472.

379 Rescorla L. 2009. Age 17 language and reading outcomes in late-talking toddlers:

380 Support for a dimensional perspective on language delay. Journal of Speech,

$381 \quad$ Language, and Hearing Research 52:16-30.

382 Rescorla L. 2011. Late talkers: Do good predictors of outcome exist? Developmental 383 Disabilities Research Reviews 17:141-150.

384 Rice ML, Taylor CL, Zubrick SR. 2008. Language outcomes of 7-year-old children 385 with or without a history of late language emergence at 24 months. Journal of Speech, Language, and Hearing Research 51:394-407.

387 Roulstone S, Law J, Rush R, Clegg J, Peters T. 2011. Investigating the role of language in children's early educational outcomes: An analysis of data from the Avon Longitudinal Study of Parents and Children (ALSPAC). Nottingham,

Scarborough HS, Dobrich W. 1990. Development of children with early language UK: Department of Education. delay. Journal of Speech and Hearing Research 33:70-83.

Semel E, Wiig E, Secord W. 2000. Clinical Evaluation of Language Fundamentals (CELF-III UK). London: Psychological Corporation. 
395 Snowling MJ, Stothard SE, Clarke P, Bowyer-Crane C, Harrington A, Truelove E, Nation K, Hulme C. 2009. York Assessment of Reading for Comprehension. London: GL Assessment.

398 Wagner R, Torgesen JK, Rashotte CA. 1999. Comprehensive Test of Phonological Processes. Austin, TX: Pro-Ed.

400 Wake M, Tobin S, Girolametto L, Ukomunne OC, Gold L, Levickis P, Sheehan J, 401 Goldfeld S, Reilly S. 2011. Outcomes of population based language promotion 402 for slow to talk toddlers at ages 2 and 3 years: Let's learn Language cluster randomised controlled trial. British Medical Journal 343:d4741.

404 Zambrana IM, Pons F, Eadie P, Ystrom E. 2014. Trajectories of language delay from 405 406 age 3 to 5: persistence, recovery and late onset. International Journal of Language and Communication Disorders 49:304-316. 


\section{Table 1 (on next page)}

Comparison of late talkers and average talkers at $\mathrm{t} 1$ 
Table 1: Comparison of average talkers (ATs) and late talkers (LTs) on vocabulary knowledge at $\mathrm{t} 1$, and demographic factors at $\mathrm{t} 1$ and $\mathrm{t} 2$, with strict and lax definition of LTs

\begin{tabular}{llllllll}
\hline Measure & LT definition & $\mathrm{N}$ & AT Mean (SD) & LT Mean (SD) & $t$ & $p$ & $d$ \\
\hline OCDI Comprehension t1 & $\leq 10$ words & 30 & $174.7(65.74)$ & $147.0(7.72)$ & 1.38 & .178 & 0.41 \\
& $\leq 6$ words & 20 & $190.6(61.65)$ & $147.1(65.29)$ & 1.87 & .077 & 0.68 \\
OCDI Production t1 $^{\mathrm{a}}$ & $\leq 10$ words & 30 & $34.77(13.38)$ & $5.20(3.21)$ & - & - & 3.04 \\
& $\leq 6$ words & 20 & $33.30(11.55)$ & $3.45(2.33)$ & - & - & 3.58 \\
Age t1 (months) & $\leq 10$ words & 30 & $18.30(0.47)$ & $18.23(0.43)$ & 0.70 & .489 & 0.16 \\
& $\leq 6$ words & 20 & $18.25(0.44)$ & $18.15(0.37)$ & 1.00 & .330 & 0.25 \\
IMD Index t1 & $\leq 10$ words & 26 & $24900(4911)$ & $24985(6181)$ & -0.06 & .954 & -0.02 \\
& $\leq 6$ words & 17 & $24914(5309)$ & $25692(4805)$ & -0.62 & .546 & -0.15 \\
Age t2 (years;months) & $\leq 10$ words & 30 & $7 ; 01(1 ; 05)$ & $7 ; 01(1 ; 04)$ & - & - & 0.02 \\
& $\leq 6$ words & 20 & $6 ; 11(1 ; 03)$ & $6 ; 11(1 ; 04)$ & - & - & 0.01 \\
IMD Index t2 & $\leq 10$ words & 27 & $24288(5910)$ & $22117(7779)$ & 1.12 & .272 & 0.31 \\
& $\leq 6$ words & 18 & $24620(6388)$ & $23322(7584)$ & 0.59 & .566 & 0.19 \\
\hline
\end{tabular}

\footnotetext{
${ }^{\text {a}}$ Variable used to define non-overlapping LT and AT groups; ${ }^{b}$ Variable used to match groups
} 


\section{Table 2 (on next page)}

Language, literacy and nonverbal abilities at t2 
Table 2: Language, literacy and nonverbal measures at $\mathrm{t} 2$ for full sample of Average Talkers (ATs) and Late Talkers (LTs), with strict and lax definitions of LT

\begin{tabular}{|c|c|c|c|c|c|c|c|}
\hline Measure & LT definition & $\mathrm{N}$ & AT Mean (SD) & LT Mean (SD) & $\mathrm{t}$ & $\mathrm{p}$ & $\mathrm{d}$ \\
\hline \multirow[t]{2}{*}{ Receptive vocabulary ${ }^{a}$} & $\leq 10$ words & 30 & $116.77(11.15)$ & $112.97(12.01)$ & 1.33 & .194 & 0.33 \\
\hline & $\leq 6$ words & 20 & $117.95(11.50)$ & $107.25(16.07)$ & 1.41 & .176 & 0.50 \\
\hline \multirow[t]{2}{*}{ Expressive vocabulary ${ }^{\mathrm{a}}$} & $\leq 10$ words & 30 & $113.67(13.01)$ & $105.60(16.18)$ & 2.13 & .042 & 0.58 \\
\hline & $\leq 6$ words & 20 & $119.95(9.60)$ & $114.85(12.77)$ & 2.36 & .029 & 0.82 \\
\hline \multirow[t]{2}{*}{ Nonword repetition $^{\mathrm{a}}$} & $\leq 10$ words & 30 & $116.38(17.43)$ & $111.54(15.23)$ & 1.24 & .224 & 0.33 \\
\hline & $\leq 6$ words & 18 & $119.44(15.43)$ & $112.50(15.59)$ & 1.57 & .132 & 0.57 \\
\hline \multirow[t]{2}{*}{ Recalling sentences ${ }^{b}$} & $\leq 10$ words & 26 & $10.33(2.00)$ & $9.89(2.68)$ & 1.02 & .315 & 0.27 \\
\hline & $\leq 6$ words & 18 & $10.89(1.94)$ & $9.94(2.84)$ & 1.79 & .091 & 0.58 \\
\hline \multirow[t]{2}{*}{ Phonological elision ${ }^{b}$} & $\leq 10$ words & 30 & $10.92(2.38)$ & $11.46(3.00)$ & -0.52 & .605 & -0.14 \\
\hline & $\leq 6$ words & 18 & $10.78(2.69)$ & $11.89(3.12)$ & -0.60 & .555 & -0.22 \\
\hline \multirow[t]{2}{*}{ Reading accuracy ${ }^{a}$} & $\leq 10$ words & 27 & $109.70(16.16)$ & $108.04(15.84)$ & 0.71 & .482 & 0.17 \\
\hline & $\leq 6$ words & 18 & $111.94(16.56)$ & $109.56(14.89)$ & 0.80 & .434 & 0.27 \\
\hline \multirow[t]{2}{*}{ Reading comprehension ${ }^{\mathrm{a}}$} & $\leq 10$ words & 27 & $114.08(7.10)$ & $111.84(9.22)$ & 0.95 & .352 & 0.25 \\
\hline & $\leq 6$ words & 16 & $115.69(5.76)$ & $112.94(9.45)$ & 1.28 & .221 & 0.46 \\
\hline \multirow[t]{2}{*}{ Nonverbal IQ ${ }^{C}$} & $\leq 10$ words & 27 & $55.15(8.19)$ & $56.74(10.28)$ & -0.46 & .651 & -0.13 \\
\hline & $\leq 6$ words & 18 & $54.83(7.70)$ & $55.17(11.07)$ & -0.12 & .909 & -0.04 \\
\hline
\end{tabular}

Note: Standardised score means shown here to allow comparison with norms; t-tests were performed on age-residualised raw scores (see text). 


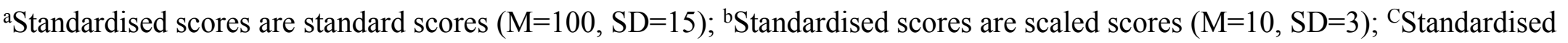
scores are $\mathrm{T}$ scores $(\mathrm{M}=50, \mathrm{SD}=10)$ 


\section{Table $\mathbf{3}$ (on next page)}

Language and literacy at t2 for TD vs. SLI 
2

Table 3: Language, literacy and nonverbal scores at $t 2$ for the subsample, grouped by lałguage status at 4 years of age (Typical Development (TD) vs. Specific Language Inspairment (SLI))

\begin{tabular}{|c|c|c|c|c|c|c|}
\hline Measure & Group & $\mathrm{N}$ & $\begin{array}{l}\text { Standardised } \\
\text { score (SD) }\end{array}$ & $t$ & $p$ & $d$ \\
\hline \multirow[t]{2}{*}{ Receptive vocabulary ${ }^{\mathrm{a}}$} & TD & 12 & $119.17(11.50)$ & 2.03 & .060 & 1.04 \\
\hline & SLI & 6 & $109.00(7.38)$ & & & \\
\hline \multirow[t]{2}{*}{ Expressive vocabulary ${ }^{\mathrm{a}}$} & TD & 12 & $116.42(7.70)$ & 5.06 & $<.001$ & 2.30 \\
\hline & SLI & 6 & $96.67(9.46)$ & & & \\
\hline \multirow[t]{2}{*}{ Nonword repetition $^{\mathrm{a}}$} & TD & 12 & $120.38(7.50)$ & 4.22 & .001 & 1.95 \\
\hline & SLI & 6 & $81.75(15.44)$ & & & \\
\hline \multirow[t]{2}{*}{ Recalling sentences ${ }^{b}$} & TD & 12 & $10.08(1.08)$ & 4.45 & $<.001$ & 2.24 \\
\hline & SLI & 6 & $7.67(1.21)$ & & & \\
\hline \multirow[t]{2}{*}{ Phonological elision } & TD & 12 & $12.00(2.14)$ & 1.82 & .088 & 0.92 \\
\hline & SLI & 6 & $8.67(1.86)$ & & & \\
\hline \multirow[t]{2}{*}{ Reading accuracy ${ }^{\mathrm{a}}$} & TD & 12 & $117.67(8.16)$ & 5.24 & $<.001$ & 2.19 \\
\hline & SLI & 6 & $91.50(13.13)$ & & & \\
\hline \multirow[t]{2}{*}{ Reading comprehension ${ }^{\mathrm{a}}$} & TD & 12 & $115.75(5.38)$ & 3.48 & .003 & 1.71 \\
\hline & SLI & 6 & $107.67(6.28)$ & & & \\
\hline \multirow[t]{2}{*}{ Nonverbal IQ ${ }^{c}$} & TD & 12 & $57.92(6.26)$ & 2.01 & .062 & 0.94 \\
\hline & SLI & 6 & $51.50(7.56)$ & & & \\
\hline
\end{tabular}

N6te: Raw scores were corrected for age at $t 2 ; t$-tests were performed on the ageregressed scores 
aSsandardised scores are standard scores $(\mathrm{M}=100, \mathrm{SD}=15)$; ${ }^{\mathrm{b}}$ Standardised scores are scaled scores $(M=10, S D=3)$; ${ }^{c}$ Standardised scores are $T$ scores $(M=50, S D=10)$ 